\title{
Review
}

\author{
C. W. S. To \\ D. M. Li \\ Department of Mechanical \\ Engineering \\ University of Western Ontario \\ London, Ontario N6A 5B9, \\ Canada
}

\section{Largest Lyapunov Exponents and Bifurcations of Stochastic Nonlinear Systems}

Two commonly adopted expressions for the largest Lyapunov exponents of linearized stochastic systems are reviewed. Their features are discussed in light of bifurcation analysis and one expression is selected for evaluating the largest Lyapunov exponent of a linearized system. An independent method, developed earlier by the authors, is also applied to determine the bifurcation points of a van der Pol oscillator under parametric random excitation. It is shown that the bifurcation points obtained by the independent technique agree qualitatively and quantitatively with those evaluated by using the largest Lyapunov exponent of the linearized oscillator. (C) 1996 John Wiley \& Sons, Inc.

\section{INTRODUCTION}

Various techniques for bifurcation analysis of nonlinear oscillators under stationary stochastic excitations exist in the literature (Boxler and Arnold, 1986). Mathematically, the techniques (Boxler, 1988; Ariaratnam and Xie, 1988) based on the largest Lyapunov exponents of linearized oscillators seem to be the most rigorous ones. However, the largest Lyapunov exponent of a linearized oscillator provides only the necessary condition for bifurcation analysis of nonlinear oscillators and therefore, additional tools are required. The technique based on using the largest Lyapunov exponent of the linearized system and the stochastic center manifold theorem requires the determination of the stochastic center manifold (Boxler, 1989). The latter, in turn, calls for the computation of the cocycle of nonlinear diffeomorphisms, which remains the major difficulty for general 2- (and higher) dimensional problems.
On the other hand, the technique based on the largest Lyapunov exponent and the method of stochastic averaging of Khas'minskii (1967) applied to two first-order equations describing the response and energy of the oscillator assumes the existence of stationary probability density distribution (Ariaratnam and Xie, 1988).

The objectives of the investigation reported here are thus twofold. First, the two expressions for the largest Lyapunov exponent presented by Ariaratnam and Xie (1988) and Pardoux and Wihstutz (1988) are reviewed. Their features are discussed in light of bifurcation analysis and the expression proposed by Pardoux and Wihstutz (1988) is selected for determining the largest Lyapunov exponent of a linearized system. The determined largest Lyapunov exponent is then applied together with the stochastic center manifold theorem to the bifurcation analysis in which the bifurcation points are located. Second, an independent method developed earlier by To et al. (1989),

Received January 22, 1995; Accepted January 10, 1996. 
which is based on a combination of the perturbation method, the limit theorem of Khas'minskii (1967), and some results from the singularity theory and group theory, is employed for the bifurcation analysis of a single degree of freedom (SDOF) system, the van der Pol oscillator, under a stationary stochastic parametric excitation. It is found that the bifurcation points obtained by the method of To and coworkers (1989) agree qualitatively with those determined by using the largest Lyapunov exponent evaluated by the expression of Pardoux and Wihstutz (1988) with the stochastic center manifold theorem of Boxler (1989). Quantitatively, the values determined by the technique of To et al. (1989) are bifurcation points for the $k$ th moment stability of the given nonlinear system, while those evaluated with the equations for the largest Lyapunov exponents given by Pardoux and Wihstutz (1988) are the bifurcation conditions for the almost sure stability of the linearized system.

\section{LARGEST LYAPUNOV EXPONENTS}

Within the domain of nonlinear dynamic systems and structural dynamics, it seems that the most commonly adopted expression for the largest Lyapunov exponent is that presented by Pardoux and Wihstutz (1988). The expressions given by the latter seem to be more user-friendly in the sense that simple and general explicit expressions are available for the determination of the largest Lyapunov exponent and corresponding rotation number of the 2-dimensional linear stochastic system with small diffusion. We introduce briefly some results of Pardoux and Wihstutz (1988) as a theorem and apply these results later in this article.

Consider a linear stochastic Stratonovich (1963) equation,

$$
d X=A X d t+\sqrt{\varepsilon} \sigma \sum_{k=1}^{r} B_{k} X d w_{k}, \quad X \in R^{2},
$$

where

$$
0<\varepsilon \ll 1, \quad \sigma \in R^{1}, A, B_{k} \quad(k=1,2, . ., r),
$$

in which $A$ and $B_{k}$ are all $2 \times 2$ real matrices and $\left\{w_{k}(t), t \geq 0, k=1,2, \ldots ., r\right\}$ are mutually independent real valued Wiener processes on a probability space with filtration $\left\{\Omega, F, F_{t}, P\right\}$. Then we have the following theorem.
THEOREM 1. (i) If the system parameter matrix

$$
A=\left[\begin{array}{rr}
a & -b \\
b & a
\end{array}\right], \quad a, b \in R, \quad b \neq 0,
$$

the largest Lyapunov exponent for system (1) is

$$
\begin{aligned}
\lambda_{\varepsilon}= & a+\frac{\varepsilon}{8} \sigma^{2} \sum_{k=1}^{r}\left[\left(b_{11}^{k}-b_{22}^{k}\right)^{2}\right. \\
& \left.+\left(b_{12}^{k}+b_{21}^{k}\right)^{2}\right]+o(\varepsilon) .
\end{aligned}
$$

(ii) If the system parameter matrix

$$
A=\left[\begin{array}{cc}
a_{1} & 0 \\
0 & a_{2}
\end{array}\right], \quad a_{2}<a_{1}, \quad a_{1}, a_{2} \in R,
$$

the largest Lyapunov exponent for system (1) is

$$
\lambda_{\varepsilon}=a_{1}+\frac{\varepsilon}{2} \sigma^{2} \sum_{k=1}^{r} b_{12}^{k} b_{21}^{k}+o(\varepsilon),
$$

where

$$
B_{k}=\left[\begin{array}{ll}
b_{11}^{k} & b_{12}^{k} \\
b_{21}^{k} & b_{22}^{k}
\end{array}\right]
$$

In the foregoing if matrices $A$ and $B_{k}$ are functions of a real parameter $\lambda$, Eqs. (2) and (3) still hold and $a, b_{i j}^{k}(i, j=1,2)$ are to be replaced by $a(\lambda)$ and $b_{i j}^{k}(\lambda)$, respectively. Moreover, Eq. (2) is associated with the corresponding deterministic system that has two complex eigenvalues, whereas Eq. (3) is concerned with the deterministic system having two distinct real eigenvalues. It is appropriate to point out that when $\varepsilon$ is not small and $\varepsilon \sigma$ approaches infinity, the largest Lyapunov exponents can be evaluated by using the expressions of Arnold and colleagues (1986). For brevity, Eqs. (2) and (3) do not include higher order terms because they are not required in the present investigation and they can be found in the references quoted above.

The second commonly adopted expression for the largest Lyapunov exponent as given by Ariaratnam and Xie (1988) is that based on a procedure due to Khas'minskii (1967) and Kozin and Prodromou (1971). It is hinged on the assumption that stationary probability density distribution exists for the two first-order equations describing the response and energy of the oscillator. The equa- 
tion of motion in this case is a linear stochastic Ito's equation

$$
d X=A X d t+\sqrt{\varepsilon} \sigma B X d w, \quad X \in R^{2},
$$

where the system parameter matrix $A$ is different from that in Eq. (1) and $w$ is a "unit" Wiener process. The results of Ariaratnam and Xie (1988) are summarized as a theorem in the following.

THEOREM 2. If parameter matrix $A$ and the forcing matrix $B$ in system (4) are given by

$$
A=\left[\begin{array}{cc}
0 & 1 \\
-\gamma^{\prime} & 0
\end{array}\right], \quad B=\left[\begin{array}{cc}
0 & 0 \\
0 & -1
\end{array}\right],
$$

the largest Lyapunov exponent for system (4) is

$$
\lambda_{x}=\frac{\varepsilon \sigma^{2}}{\left[2\left(\sqrt{\gamma^{\prime}}+1\right)^{2}\right]}, \quad \gamma^{\prime}>0,
$$

and

$$
\lambda_{x}=\sqrt{-\gamma^{\prime}}+\frac{\varepsilon \sigma^{2}\left(1+\gamma^{\prime}\right)}{\left[2\left(1-\gamma^{\prime}\right)^{2}\right]}, \quad \gamma^{\prime}<0 .
$$

When $\left|\gamma^{\prime}\right|=0$ the parameter matrix $A$ of system (4) has two eigenvalues equal to zero. That is, the matrix $A$ is nilpotent. This is the case not covered by Eqs. (2) and (3), however, this case is considered by Wihstutz (1986). The difference between the largest Lyapunov exponent of Pardoux and Wihstutz (1988) and Ariaratnam and Xie (1988) is that the former explicit expressions were given in series form, whereas the expression of Ariaratnam and Xie (1988) contains only two terms that correspond to the zeroth- and firstorder terms of Pardoux and Wihstutz (1988). Furthermore, as pointed out earlier, the expression of Ariaratnam and Xie (1988) requires the existence of stationary probability density distribution whereas no such assumption is necessary in the expression of Pardoux and Wihstutz (1988). Clearly, the largest Lyapunov exponents given by Eqs. (2) and (3) are for systems different from those governed by Eqs. (5a) and (5b).

\section{APPLICATIONS OF LARGEST LYAPUNOV EXPONENTS}

As an illustration to the application of Eqs. (2) and (3), the following oscillator is considered:

$$
\ddot{x}+\left[\gamma^{\prime}+\sqrt{\varepsilon} \sigma \xi(t)\right] x=0,
$$

where $\xi(t)$ is the parametric white noise excitation and the double overdot represents the second derivative with respect to time $t$. The system described by Eq. (6) was studied by Ariaratnam and Xie (1988). It was pointed out by them that using the expressions of Pardoux and Wihstutz (1988) for the above system gives the largest Lyapunov exponent as

$$
\lambda_{x}=\frac{\varepsilon \sigma^{2}}{8 \gamma^{\prime}}, \quad \gamma^{\prime}>0
$$

Similarly,

$$
\lambda_{x}=\sqrt{-\gamma^{\prime}}+\frac{\varepsilon \sigma^{2}}{8 \gamma^{\prime}}, \quad \gamma^{\prime}<0 .
$$

For finite bifurcation parameter $\gamma^{\prime}$, Eqs. (7) and (8) agree with those obtained by Eq. (5). However, it was also pointed out by Ariaratnam and Xie (1988) that Eqs. (7) and (8) are invalid when $\left|\gamma^{\prime}\right|$ approaches zero. Superficially, it seems that the finding of Ariaratnam and Xie (1988) is correct and that the expression for the determination of the largest Lyapunov exponent summarized in Theorem 2 is superior to those in Theorem 1. It should be noted that when $\left|\gamma^{\prime}\right|=0$ the parameter matrix $A$ of the system has two eigenvalues equal to zero; and, as pointed out earlier, the definitions in Eqs. (2) and (3) cannot be employed.

To further reveal the features of the largest Lyapunov exponents expressed in Theorem 1, we propose to study them in light of bifurcation analysis and we confine the study to $\left|\gamma^{\prime}\right|$ other than zero. To this end the following is in order.

It was stated by Boxler (1988) that the necessary condition for bifurcation of nonlinear systems to occur is that the largest Lyapunov exponent equals zero. Applying this condition to Eq. (7) for system (6) one has

$$
\sigma=0 \text { for } \gamma^{\prime}>0 .
$$

Equation (9) implies that no stochastic excitation is applied to the oscillator and therefore the possible bifurcation point is that of the deterministic system.

Similarly, applying the condition of zero largest Lyapunov exponent to Eq. (8) one obtains

$$
8 \gamma^{\prime} \sqrt{-\gamma^{\prime}}=-\varepsilon \sigma^{2} \text { for } \gamma^{\prime}<0 .
$$


If $\gamma^{\prime}=0$, Eq. (10) gives $\sigma=0$, which implies that the possible bifurcation point is the deterministic one. Consequently, Eq. (7) and (8) for the system described by Eq. (6) are correct and logical.

However, up to this stage nothing is revealed in regard to the location of the bifurcation point of system (6). Therefore, we consider the system described by Eq. (6) with transformation $q=x$ $\exp (-\beta t)$ for $\beta>0$. This transformation for system (6) was adopted by Ariaratnam and Xie (1988) and Arnold et al. (1986). By making use of this transformation, it can be shown that the following equation reduces to Eq. (6):

$$
\ddot{q}+2 \beta \dot{q}+[-\gamma+\sqrt{\varepsilon} \sigma \xi(t)] q=0,
$$

where $\gamma^{\prime}=-\gamma-\beta^{2}$ was applied. It may be appropriate to mention that when $\left|\gamma^{\prime}\right|=0$ this means that system (11) has two equal real eigenvalues. This case is not covered by Pardoux and Wihstutz (1988) but was considered by Wihstutz (1986).

Now, if $\gamma^{\prime}$ is small and because it is given in Eq. (10) that $\gamma^{\prime}<0$, we can write this value as

$$
\gamma^{\prime}=-\gamma^{*},
$$

where $\gamma^{*}$ is a positive value. Recall that $\gamma^{\prime}=$ $-\gamma-\beta^{2}$ in Eq. (11). Hence, $\gamma=\gamma^{*}-\beta^{2}$. If we plot the response $q$ versus the bifurcation parameter $\gamma$ to determine the bifurcation points, then when we set $\gamma=0$ we have $\gamma^{*}=\beta^{2}$. This implies that if we plot $q$ versus $\gamma$ the possible stochastic bifurcation point $\gamma^{*}$ is positive and therefore is on the right-hand side (RHS) of $\gamma=0$, because $\beta>0$. When $\gamma=0$ and $\sigma=0$ then $\gamma^{*}$ is the deterministic bifurcation point. Thus, for $\sigma \neq 0$, $\gamma^{*}$ is on the RHS of the deterministic bifurcation point. These results make physical sense but are not so direct in that they are derived by the combined use of Eqs. (11) and (10) that, in turn, makes use of Eq. (8) that was originated from system (6). Alternatively and more directly, we assume that $\gamma \geq 0$ and applying Eq. (3) to system (11) we can show that

$$
\lambda_{\varepsilon}(\gamma)=-\beta+\sqrt{\beta+\gamma}-\frac{\varepsilon \sigma^{2}}{8\left(\beta^{2}+\gamma\right)}+o\left(\varepsilon \sigma^{2}\right) .
$$

Thus, for $\gamma=0$ we have

$$
\lambda_{\varepsilon}(0)=-\frac{\varepsilon \sigma^{2}}{8 \beta^{2}}+o\left(\varepsilon \sigma^{2}\right)<0 .
$$

For $\gamma=1$, Eq. (3) gives

$$
\begin{aligned}
\lambda_{\varepsilon}(1)= & -\beta+\sqrt{\beta+1} \\
& -\frac{\varepsilon \sigma^{2}}{8\left(\beta^{2}+1\right)}+o\left(\varepsilon \sigma^{2}\right)>0 .
\end{aligned}
$$

Conditions (13) and (14) implies that there is a value of $\gamma$, say $\gamma^{0}$, such that

$$
\lambda_{\varepsilon}\left(\gamma^{0}\right)=0, \quad 0<\gamma^{0}<1 .
$$

That is, $\gamma^{0}$ is positive. When the deterministic system of Eq. (11), that is, when $\sigma=0$, undergoes a bifurcation at $\gamma=0$, then the stochastic bifurcation point $\gamma^{0}$ is on the RHS of $\gamma=0$. This finding agrees with that found by using Eq. (8) and it is not surprising because both the largest Lyapunov exponents determined above are from Eq. (3).

It should be noted that although the stochastic center manifold of Boxler (1989) requires no limitation on the size of noise intensity, the largest Lyapunov exponent expressions of Pardoux and Wihstutz (1988) and that given by Ariaratnam and Xie (1988) do assume small noise intensity. The implication of this is that even using the stochastic center manifold for nonlinear systems in conjunction with the largest Lyapunov exponent, evaluated by either one of the foregoing techniques for the bifurcation analysis, one can only deal with small noise intensity. As pointed out by To (1993), to deal with the bifurcation analysis of a nonlinear oscillator excited by noise of large intensity, one has to determine the largest Lyapunov exponent of the linearized oscillator by employing the results of Arnold et al. (1986) in addition to the application of the stochastic center manifold theorem.

In the following we show that the bifurcation points evaluated by an independent method developed earlier by To et al. (1989) agree qualitatively with those determined by using the largest Lyapunov exponent in Eq. (2). The technique of To and coworkers (1989) consists of the theorem of Hopf bifurcation, stochastic averaging, some results from the singularity theory, and group theory.

\section{BIFURCATION ANALYSIS OF NONLINEAR STOCHASTIC SYSTEMS}

Consider the parametrically excited stochastic van der Pol oscillator 


$$
\ddot{x}-\lambda \dot{x}+\alpha x^{2} \dot{x}+x=\sqrt{\varepsilon} \xi(t) x,
$$

where $\lambda$ is a small positive parameter and $\alpha$ is a positive parameter of order 1 , the overdot designates the derivative with respect to time $t$, and the remaining symbols are as defined in the foregoing sections.

Before the determination of the bifurcation points of the above system, we outline briefly the steps required and cast it in a form similar to that in To et al. (1989). First, Eq. (15) is written in the state space or vector space form such that

$$
\begin{aligned}
\frac{d y}{d t} & =F(y, \lambda)+\sqrt{\varepsilon}\left(A_{r} f_{r}(t)\right) y, \\
r & =1,2, \ldots, n,
\end{aligned}
$$

where $y=\left[\begin{array}{ll}y_{1} & y_{2}\end{array}\right]^{T}, y_{1}=x, y_{2}=d x / d t, \lambda$ is the bifurcation parameter, and $A_{r}=A_{r}(\lambda)$ are constant $2 \times 2$ matrices that are $C^{\infty}$ in $\lambda$; and $f_{r}(t)$ are uncorrelated broadband stationary stochastic processes with zero mean values and have arbitrary smoothly varying spectral density function. Expanding $F(y, \lambda)$ in a Taylor's series about the point $(0,0)$, representing the second and third homogeneous polynomials as $H_{2}(y)$ and $H_{3}(y)$, respectively, and disregarding the excitation terms at this stage, one has

$$
\begin{aligned}
\frac{d y}{d t}= & A y+\lambda\left[\begin{array}{cc}
\sigma^{\prime}(0) & \omega^{\prime}(0) \\
-\omega^{\prime}(0) & \sigma^{\prime}(0)
\end{array}\right] y+H_{2}(y) \\
& +H_{3}(y)+o\left(y^{3}, \lambda\right)
\end{aligned}
$$

where the system parameter matrix $A=A(0)$ is a matrix of order 2 . This is different from the coefficient matrices of the forcing function, $A_{r}$. Also, $\sigma(\lambda) \pm i \omega(\lambda)$ are the eigenvalues of $A(\lambda)$,

$$
\begin{aligned}
& H_{2}(y)=\left\{\begin{array}{l}
B_{i j}^{(1)} y_{i} y_{j} \\
B_{i j}^{(2)} y_{i} y_{j}
\end{array}\right\}_{i \leq j} \quad(i, j=1,2), \\
& H_{3}(y)=\left\{\begin{array}{l}
C_{i j k}^{(1)} y_{i} y_{j} y_{k} \\
C_{i j k}^{(2)} y_{i} y_{j} y_{k}
\end{array}\right\}_{i \leq j \leq k} \quad(i, j, k=1,2),
\end{aligned}
$$

in which $B_{i j}^{(1)}, B_{i j}^{(2)}, C_{i j k}^{(1)}$, and $C_{i j k}^{(2)}$ are constants. It should be noted that the symbol $\sigma(\lambda)$ here is different from $\sigma$ in Eqs. (1)-(14).

Second, we want to obtain the so-called standard form equation. To this end, we rescale the state vector $y$ by setting

$$
\begin{array}{rlrl}
z & =\left[\begin{array}{ll}
z_{1} & z_{2}
\end{array}\right]^{T}, & z_{1}=\sqrt{\varepsilon} y_{1}, \\
z_{2}=\sqrt{\varepsilon} y_{2}, & \lambda=\sqrt{\varepsilon} \eta,
\end{array}
$$

in which the superscript $T$ designates the transpose. Then by making use of Eq. (16) we have

$$
\begin{aligned}
\frac{d z}{d t}= & A z+\varepsilon \eta\left[\begin{array}{cc}
\sigma^{\prime}(0) & \omega^{\prime}(0) \\
-\omega^{\prime}(0) & \sigma^{\prime}(0)
\end{array}\right] z+\sqrt{\varepsilon} H_{2}(z) \\
& +\varepsilon H_{3}(z)+\sqrt{\varepsilon}\left(A_{r} f_{r}(t)\right)+o(\varepsilon),
\end{aligned}
$$

where

$$
A_{r}(0)=\left[\begin{array}{ll}
A_{r 11} & A_{r 12} \\
A_{r 21} & A_{r 22}
\end{array}\right] .
$$

Mathematically, we can further apply the transformation

$$
z_{1}=a \sin \Phi, \quad z_{2}=a \cos \Phi, \quad \Phi=t+\phi,
$$

such that

$$
a^{2}=z_{1}^{2}+z_{2}^{2}, \quad \Phi=\tan ^{-1}\left(\frac{z_{1}}{z_{2}}\right) .
$$

Physically, if the damping parameter $\lambda$ in Eq. (17) is small the random amplitude $a$ and the random phase $\phi$ in Eq. (19) are "slowly varying" with respect to time $t$.

After some lengthy algebraic manipulation, we can show that

$$
\begin{aligned}
\dot{a}= & \varepsilon\left[\sigma^{\prime}(0) \eta-R_{z} a^{2}\right] a \\
& +\frac{1}{2} \sqrt{\varepsilon} a\left[\left(A_{r 11}+A_{r 22}\right)\right. \\
& +\left(A_{r 12}+A_{r 21}\right) \sin (2 \Phi) \\
& \left.+\left(A_{r 22}-A_{r 11}\right) \cos (2 \Phi)\right] f_{r}(t), \\
\dot{\phi}= & \varepsilon\left[\omega^{\prime}(0) \eta-Q_{z} a^{2}\right] \\
& +\frac{1}{2} \sqrt{\varepsilon}\left[\left(A_{r 12}-A_{r 21}\right)\right. \\
& +\left(A_{r 11}-A_{r 22}\right) \sin (2 \Phi) \\
& \left.+\left(A_{r 12}+A_{r 21}\right) \cos (2 \Phi)\right] f_{r}(t),
\end{aligned}
$$


where

$$
\begin{aligned}
R_{z}= & -\frac{1}{8}\left(2 B_{11}^{(1)} B_{11}^{(2)}-B_{11}^{(1)} B_{12}^{(1)}-B_{12}^{(1)} B_{22}^{(1)}\right. \\
& \left.+B_{11}^{(1)} B_{12}^{(1)}+B_{12}^{(2)} B_{22}^{(2)}-2 B_{22}^{(1)} B_{22}^{(2)}\right) \\
& -\frac{1}{8}\left(3 C_{111}^{(1)}+C_{112}^{(2)}+C_{122}^{(1)}+3 C_{222}^{(2)}\right)
\end{aligned}
$$

is the coefficient associated with the nonlinear term in the system while

$$
\begin{aligned}
Q_{z}= & -\frac{1}{8}\left[\left(B_{11}^{(1)}+B_{22}^{(1)}\right)\left(B_{12}^{(2)}-B_{11}^{(1)}-3 B_{22}^{(1)}\right)\right. \\
& +\left(B_{11}^{(2)}+B_{22}^{(2)}\right)\left(B_{12}^{(1)}-B_{22}^{(2)}-3 B_{11}^{(2)}\right) \\
& +\frac{1}{3}\left(B_{11}^{(1)}-B_{22}^{(1)}-B_{12}^{(2)}\right)\left(B_{22}^{(1)}-B_{11}^{(1)}+B_{12}^{(2)}\right) \\
& +\frac{1}{3}\left(B_{22}^{(2)}-B_{11}^{(2)}-B_{12}^{(1)}\right)\left(B_{11}^{(2)}-B_{22}^{(2)}+B_{12}^{(1)}\right) \\
& \left.+\left(3 C_{222}^{(1)}+C_{112}^{(1)}-C_{222}^{(2)}-3 C_{111}^{(2)}\right)\right] .
\end{aligned}
$$

Equation (20) is the required standard form and is equivalent to Eq. (15) to $o(\varepsilon)$.

We can use the stochastic averaging method for Eq. (20). Under the hypothesis that the processes $(a, \phi)$ converge weakly to diffusive Markov processes, Ito's equations are obtained. Finally, the bifurcation points for the system defined by Eq. (15) and its approximation in Eq. (20) can be found with the remaining steps presented by To et al. (1989) as

$$
\begin{aligned}
\eta_{c}= & \\
-\frac{\left[\left(A_{r 12}+A_{r 21}\right)^{2}+\left(A_{r 22}-A_{r 11}\right)^{2}\right] S_{r}(2)}{\left[4 \sigma^{\prime}(0)\right]} &
\end{aligned}
$$

where $S_{r}(2)$ is the spectral density at twice the natural frequency of the linearized system whose natural frequency is $1 \mathrm{rad} / \mathrm{s}$, and $\sigma^{\prime}(0)$ is the eigenvalue crossing speed that is not zero. Equation (21) is concerned with Hopf bifurcation. The degenerated case of $\sigma^{\prime}(0)=0$ was considered by To and Li (1991). For system (15), Eq. (21) gives

$$
\eta_{c}=-\frac{1}{2} S_{1}(2)<0
$$

because the spectral density of the wide-band stationary Gaussian excitation $S_{1}(2)$ is always positive.

By making use of equations (2.9) and (7.42) of
Stratonovich (1963) for system (15), one has $S_{1}(2)=\varepsilon / 2$.

Similarly, if $-\lambda$ in Eq. (15) is replaced by $+\lambda$ it can be shown that

$$
\eta_{c}=\frac{1}{2} S_{1}(2)>0
$$

For completeness and direct reference, the bifurcation solutions given by To and coworkers (1989), associated with Eqs. (22) and (23) are included in Fig. 1. In the latter, $\sigma^{\prime}(0)>0$ is associated with a negative linear damping coefficient, while $\sigma^{\prime}(0)<0$ is associated with a positive linear damping coefficient. The angular brackets in the figure denote ensemble average.

Now, applying the largest Lyapunov exponent expression in Eq. (2) to the system described by Eq. (15) we have

$$
\lambda_{\varepsilon}(\lambda)=\frac{\lambda}{2}+\frac{\varepsilon}{8}\left[\lambda^{2}+\frac{\left(4-2 \lambda^{2}\right)^{2}}{4\left(4-\lambda^{2}\right)}\right]+o(\varepsilon)
$$

Setting $\lambda=0$, Eq. (24) gives

$$
\lambda_{\varepsilon}(0)=\frac{\varepsilon}{8}+o(\varepsilon)>0
$$

On the other hand, for bifurcation to occur one must have $\lambda_{\varepsilon}\left(\lambda_{c}\right)=0$. To satisfy the latter condition, $\lambda_{c}$ must be negative with reference to Eqs. (24) and (25). That is, the bifurcation point $\lambda_{c}$ is left of $\lambda=0$. This is known by making use of Eq. (24) and recalling that $\lambda_{c}$ is small such that the bifurcation point in this case is $\lambda_{c}=-\varepsilon / 4$.

Similarly, if $-\lambda$ is replaced by $+\lambda$ in Eq. (15) then by Eq. (2) one has

$$
\lambda_{\varepsilon}(\lambda)=-\frac{\lambda}{2}+\frac{\varepsilon}{8}\left[\lambda^{2}+\frac{\left(4-2 \lambda^{2}\right)^{2}}{4\left(4-\lambda^{2}\right)}\right]+o(\varepsilon) .
$$

Thus, for $\lambda=0$ we have

$$
\lambda_{\varepsilon}(0)=\frac{\varepsilon}{8}+o(\varepsilon)>0 .
$$

For bifurcation to occur, $\lambda_{\varepsilon}\left(\lambda_{c}\right)=0$, one has from Eq. (26)

$$
\begin{aligned}
\lambda_{\varepsilon}\left(\lambda_{c}\right)= & 0=-\frac{\lambda_{c}}{2}+\frac{\varepsilon}{8}\left[\lambda_{c}^{2}+\frac{\left(4-2 \lambda_{c}^{2}\right)^{2}}{4\left(4-\lambda_{c}^{2}\right)}\right] \\
& +o(\varepsilon) .
\end{aligned}
$$




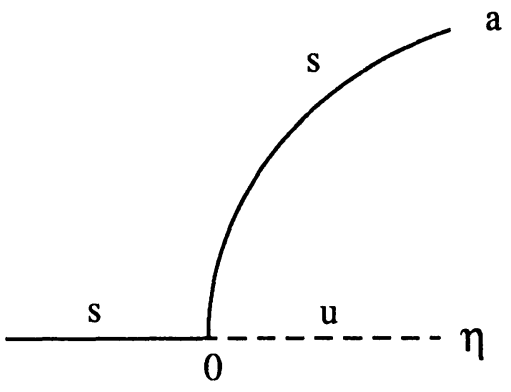

(i) Supercritical bifurcations in which

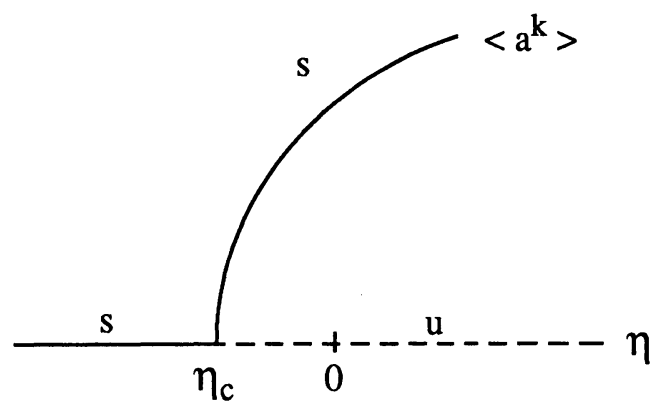

$r_{\mathrm{z}}>0, \quad \sigma^{\prime}(0)>0$.

a

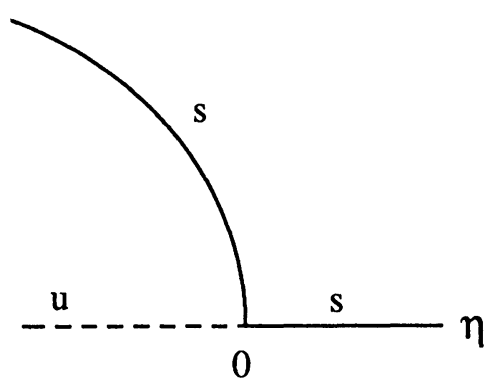

(ii) Subcritical bifurcations in which

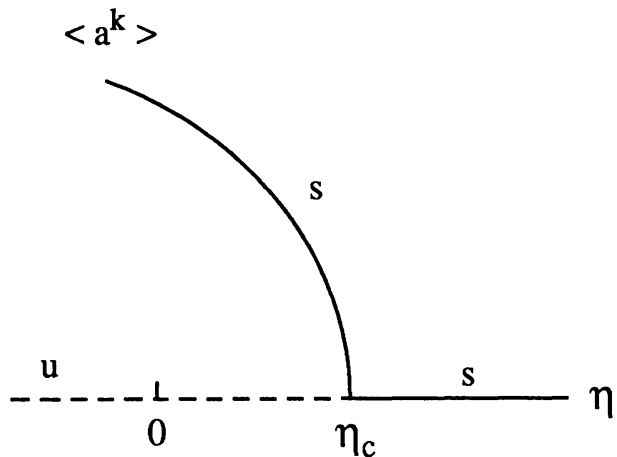

$\mathrm{r}_{\mathrm{z}}>0, \quad \sigma^{\prime}(0)<0$.

FIGURE 1 Hopf bifurcations of the single degree of freedom oscillator. $s$ and $u$ denote stable and unstable solutions, respectively.

Clearly, $\lambda_{c}$ is positive and small such that its higher power terms in Eq. (28) can be disregarded. Therefore, $\lambda_{c}$ is on the RHS of $\lambda=0$. By applying Eq. (28), the bifurcation point in this case is $\lambda_{c}=\varepsilon / 4$.

To summarize, the bifurcation point $\lambda_{c}$ determined by Eq. (28), for example, tallies qualitatively and quantitatively with that given by Eq. (23), which is obtained by an independent technique of To et al. (1989). Similarly, $\lambda_{c}$ determined by Eq. (24) agrees with (22). Clearly, the independent technique is superior to methods based on the largest Lyapunov exponent and stochastic center manifold theorem that requires the determination of the stochastic center manifold. The latter is relatively much more difficult to achieve for general 2-dimensional nonlinear systems. For higher dimensional nonlinear systems, however, techniques for determining the stochastic center manifold have yet to be developed. Note that $\eta_{c}$ by Eqs. (22) and (23) are the bifurcation points for the $k$ th moment stability of system (15), while $\lambda_{c}$ by Eqs. (24) and (28) are the bifurcation conditions for the almost sure stability of the linearized system of Eq. (15).

\section{CONCLUDING REMARKS}

By applying the definition of Pardoux and Wihstutz (1988) for the largest Lyapunov exponent of a linearized dynamic system, one can determine the stochastic bifurcation points if they exist. With an independent method based on a combination of the perturbation method, the limit theorem of Khas'minskii (1967), and some results from the singularity theory and group theory, a 
bifurcation analysis was performed on a parametrically excited stochastic van der Pol oscillator. The bifurcation points evaluated by this independent method are in agreement qualitatively and quantitatively with those obtained by using the largest Lyapunov exponent expressions of Pardoux and Wihstutz (1988) together with the stochastic center manifold theorem of Boxler (1989). In other words, the bifurcation points of a system are identical whether they are based on the $k$ th moment stability of system (15) or on the almost sure stability of the linearized system of Eq. (15). This indicates that the technique of To et al. (1989) can give correct results and is more direct in that it does not require the computation of the largest Lyapunov exponent and application of the stochastic center manifold theorem for nonlinear systems under wide band stationary stochastic excitations.

Concerning the results for the van der Pol oscillator under parametric stochastic excitation, it is found that with the linear damping coefficient $\lambda$ as the bifurcation parameter and with reference to Fig. 1, bifurcation occurs on the left-hand side of the deterministic bifurcation point, and bifurcation occurs on the RHS of the deterministic bifurcation point if $-\lambda$ in Eq. (15) is replaced by $+\lambda$.

The first author gratefully acknowledges the financial support of the Natural Sciences and Engineering Research Council of Canada for this investigation. An early version of the results was first presented at the International Conference on Stochastic Structural Dynamics, Boca Raton, FL, May 9-11, 1990.

\section{REFERENCES}

Ariaratnam, S. T., and Xie, W. C., 1988, "Stochastic Perturbation of Pitchfork Bifurcations," in Proceedings of the Symposium on Stochastic Structural Dynamics, University of Illinois at UrbanaChampaign, October 30-November, 1988, pp. 3-14. Arnold, L., Papanicolaou, G., and Wihstutz, V., 1986, "Asymptotic Analysis of the Lyapunov Exponent and Rotation Number of the Random Oscillator,"
SIAM Journal of Applied Mathematics, Vol. 46, pp. 427-450.

Boxler, P., 1988, "Lyapunov Exponents and Stochastic Center Manifolds as Tools in Stability and Stochastic Bifurcation Theory," Report No. 197, Institut für Dynamische Systeme, Universität Bremen, Bremen, Germany.

Boxler, P., 1989, "A Stochastic Version of Center Manifold Theory," Journal of Probability Theory and Related Fields, Vol. 83, pp. 509-545.

Boxler, P., and Arnold, L., 1986, "Stochastische Bifurkationstheorie," Report No. 163, Institut für Dynamische Systeme, Universität Bremen, Bremen, Germany.

Khas'minskii, R. Z., 1967, “Necessary and Sufficient Conditions for Asymptotic Stability of Linear Stochastic Systems," Journal of Theory of Probability and Its Applications, Vol. 12, pp. 144-147.

Kozin, F., and Prodromou, S., 1971, "Necessary and Sufficient Conditions for Almost Sure Sample Stability of Linear Ito Equations," SIAM Journal of Applied Mathematics, Vol. 21, pp. 413-424.

Pardoux, E., and Wihstutz, V., 1988, "Lyapunov Exponent and Rotation Number of Two-Dimensional Linear Stochastic Systems with Small Diffusion," SIAM Journal of Applied Mathematics, Vol. 48, pp. 442-457.

Stratonovich, R. L., 1963, Topics in The Theory of Random Noise, Vol. 1, Gordon and Breach Science Publishers, New York.

To, C. W. S., 1993, "'On Deterministic and Stochastic Center Manifolds," in W. Z. Chien, Z. H. Guo and Y. Z. Guo, Proceedings of the Second International Conference on Nonlinear Mechanics, Beijing, Peking University Press, August 23-26, pp. 599-602.

To, C. W. S., and Li, D. M., 1991, "Degenerated Hopf Bifurcation in a Stochastically Disturbed System," NDRL TR-9103, April 1991, Department of Mechanical Engineering, The University of Western Ontario.

To, C. W. S., Li, D. M., and Huang, K. L., 1989, "Hopf Bifurcation in a Stochastically Disturbed System," Engineering Science Preprints, ESP26.89003, 26th Annual Technical Meeting of the Society of Engineering Science, September 18-20, 1989, The University of Michigan, Ann Arbor, MI. (Also appeared as NDRL TR-9106, June 1991, Department of Mechanical Engineering, The University of Western Ontario.)

Wihstutz, V. (Ed.), 1986, Lyapunov Exponents, Lecture Notes in Mathematics, Vol. 1186, SpringerVerlag, New York. 

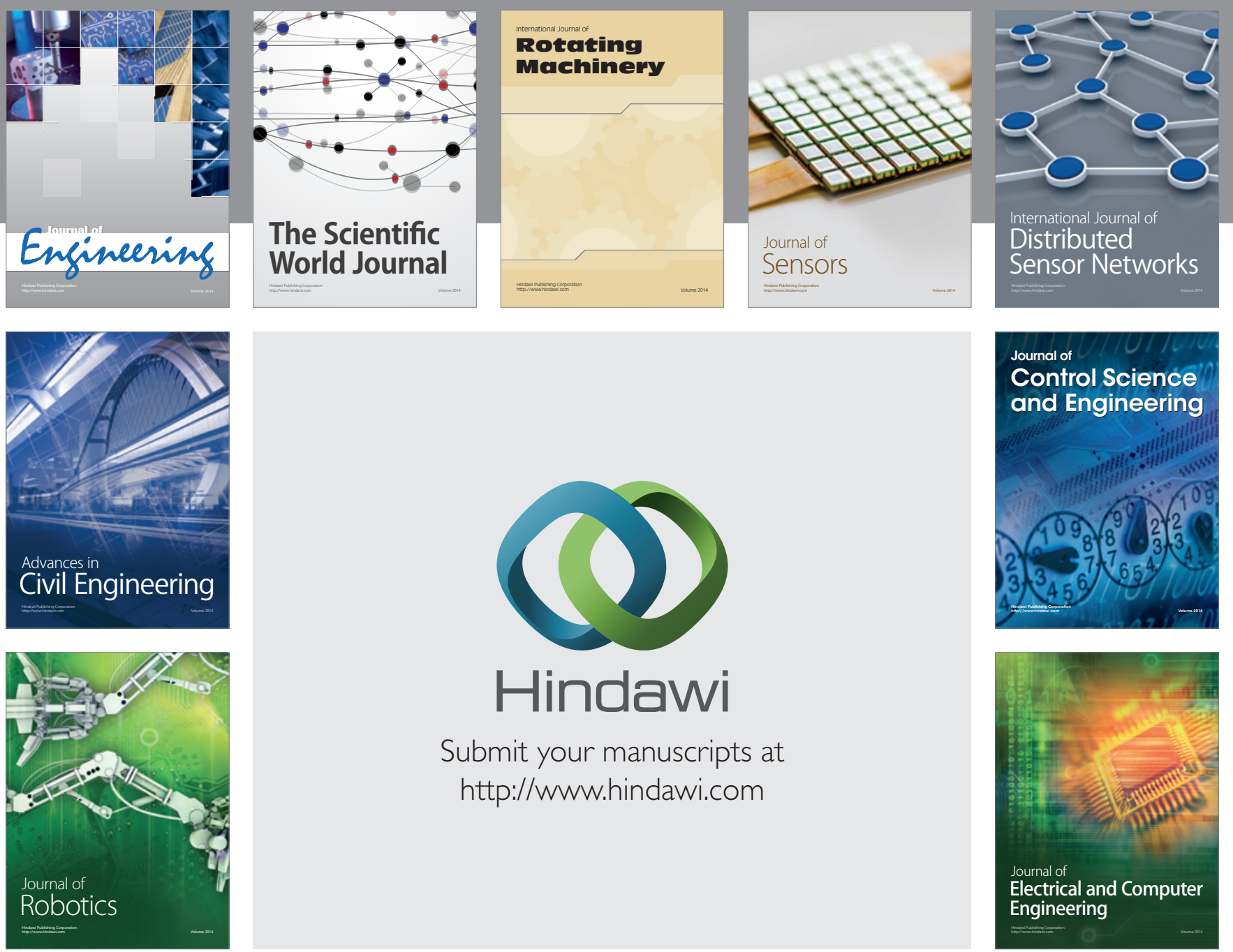

Submit your manuscripts at

http://www.hindawi.com
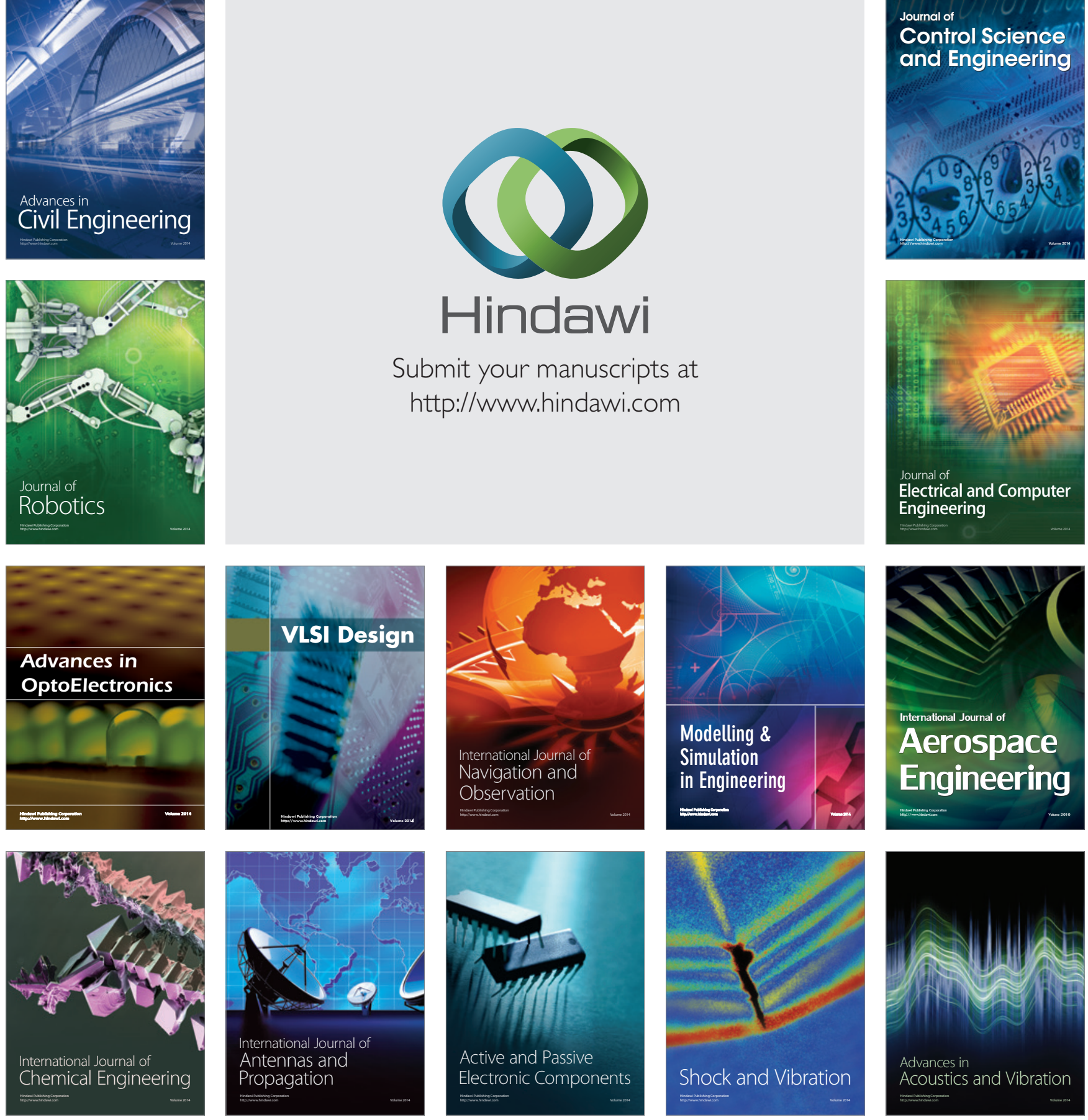